\title{
Macroscopic Supramolecular Assembly Strategy to Construct 3D Biocompatible Microenvironments with Site-Selective Cell Adhesion
}

\author{
Changyu Wang, ${ }^{\S}$ Cuiling Lin, ${ }^{\S}$ Rui Ming, Xiangxin Li, Pascal Jonkheijm, Mengjiao Cheng,* \\ and Feng Shi*
}

Cite This: ACS Appl. Mater. Interfaces 2021, 13, 28774-28781

Read Online

ACCESS

Llll Metrics \& More

Article Recommendations

Supporting Information

ABSTRACT: Three-dimensional (3D) scaffolds with chemical diversity are significant to direct cell adhesion onto targeted surfaces, which provides solutions to further control over cell fates and even tissue formation. However, the site-specific modification of specific biomolecules to realize selective cell adhesion has been a challenge with the current methods when building 3D scaffolds. Conventional methods of immersing as-prepared structures in solutions of biomolecules lead to nonselective adsorption; recent printing methods have to address the problem of switching multiple nozzles containing different biomolecules. The recently

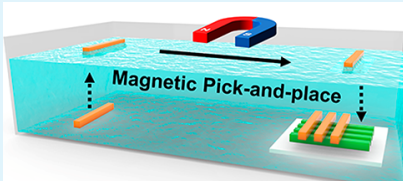

Macroscopic

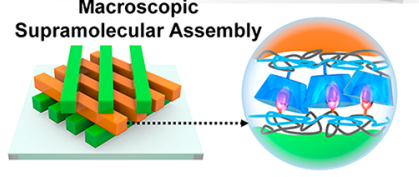

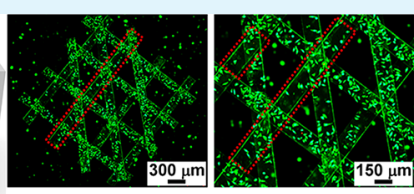

3D-Selective Cell Adhesion

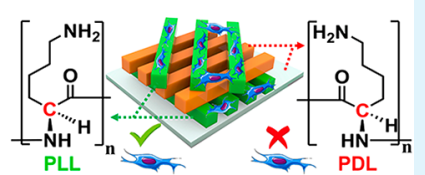
developed concept of macroscopic supramolecular assembly (MSA) based on the idea of "modular assembly" is promising to fabricate such 3D scaffolds with advantages of flexible design and combination of diverse modules with different surface chemistry. Herein we report an MSA method to fabricate 3D ordered structures with internal chemical diversity for site-selective cell adhesion. The 3D structure is prepared via 3D alignment of polydimethylsiloxane (PDMS) building blocks with magnetic pick-and-place operation and subsequent interfacial bindings between PDMS based on host/guest molecular recognition. The site-specific cell affinity is realized by distributing targeted building blocks that are modified with polylysine molecules of opposite chiralities: PDMS modified with films containing poly-L-lysine (PLL) show higher cell density than those with poly-D-lysine (PDL). This principle of selective cell adhesion directed simply by spatial distribution of chiral molecules has been proven effective for five different cell lines. This facile MSA strategy holds promise to build complex 3D microenvironment with on-demand chemical/biological diversities, which is meaningful to study cell/material interactions and even tissue formation.

KEYWORDS: macroscopic supramolecular assembly, 3D ordered structures, host-guest molecular recognition, chiral polyelectrolyte multilayer, selective cell adhesion

\section{INTRODUCTION}

Tissue engineering is a revolutionary concept used to restore the function of damaged tissues by tuning multiple properties of cells, scaffolds, and biomolecules. ${ }^{1-3}$ An ideal tissue scaffold should mimic a three-dimensional (3D) intracorporal microenvironment and exhibit controlled distribution of heterogeneous materials, biological moieties, or chemical species at designated locations to support cell growth and in vitro tissue formation. ${ }^{4}$ Such scaffolds can facilitate the site-selective adhesion of specific cells in 3D scaffolds by design for targeted cell differentiation/growth and formation of tissues. ${ }^{5-7}$ It is difficult to achieve multiple purposes of facile processability, control over 3D porous geometry, biocompatibility, and 3D site-selective cell adhesion, making the fabrication of such scaffolds a challenging process. To date, most researchers have demonstrated selective cell adhesion on two-dimensional (2D) surfaces and films ${ }^{8-11}$ by using peptides that target specific cells or patterned surface modification with different chiral molecules. ${ }^{12,13}$ Methods of constructing a 3D microenviron- ment to achieve 3D site-selective cell adhesion are still rare. However, it is important to develop facile strategies that can provide a platform for engineering targeted cells or even tissues in the future.

The macroscopic supramolecular assembly (MSA) is a new concept with the focus on mild noncovalent interactions between surfaces with a dimension larger than $10 \mu \mathrm{m},{ }^{14-18}$ which may provide solutions to building such 3D scaffolds based on the idea of modular assembly. With an MSA process, multiple materials including metals, resins and hydrogels have been built into $3 \mathrm{D}$ ordered structures, ${ }^{19-21}$ whose geometry can be well adjusted. Especially, the obtained 3D structures

Received: March 19, 2021

Accepted: June 1, 2021

Published: June 11, 2021 


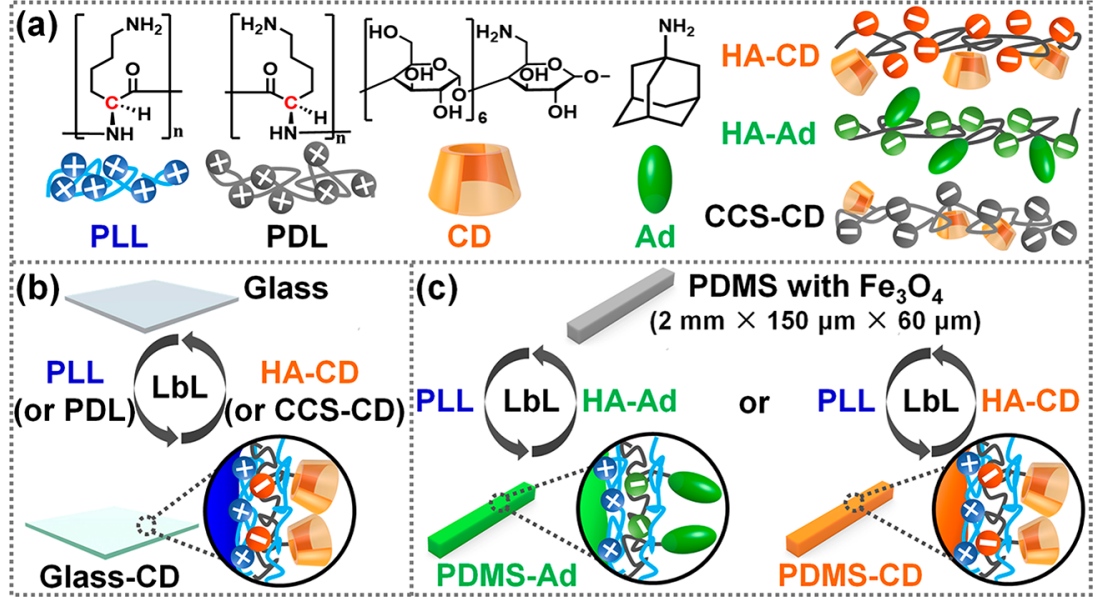

(d)

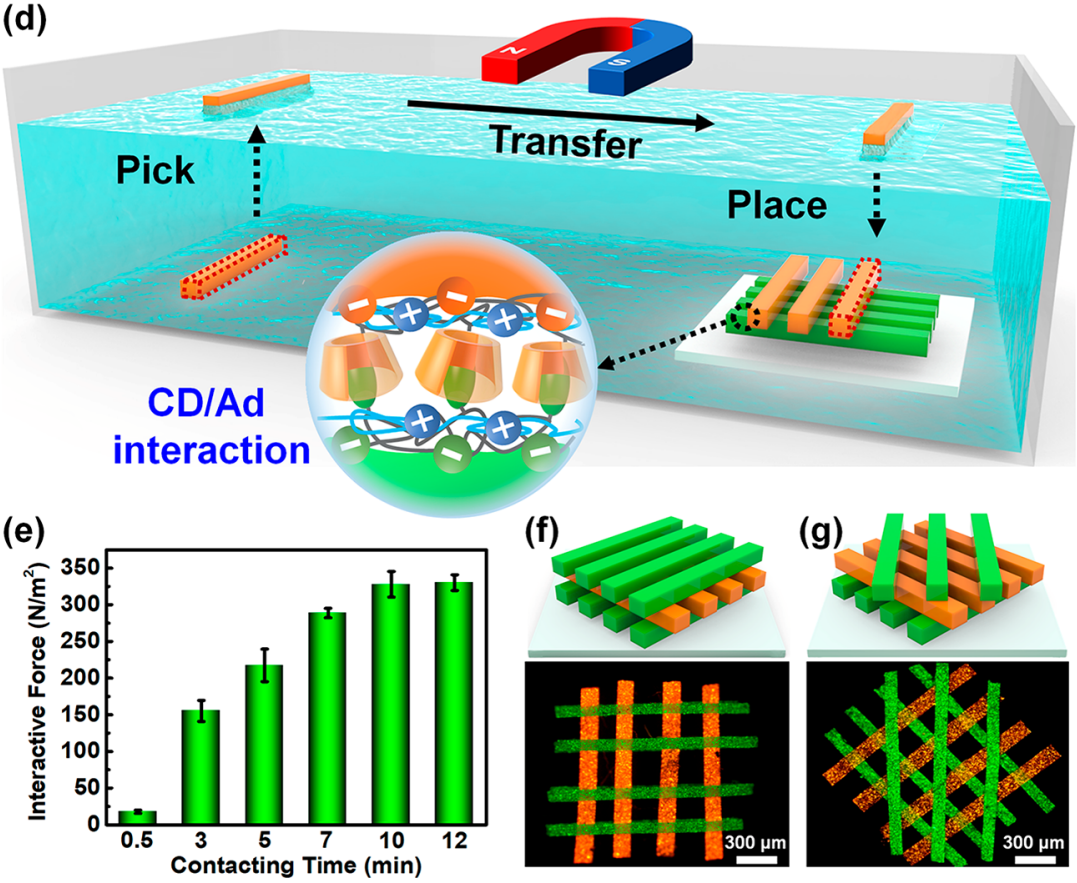

Figure 1. Schematic illustrations of (a) polyelectrolytes used for $(b, c)$ LbL surface modification, and (d) stepwise MSA to fabricate a 3D structure via the pick-and-place manipulation assisted by magnetic-field-guidance. (e) Contacting-time dependent interactive forces between the PDMS modified with (PLL/HA-CD) $)_{30.5}$ and (PLL/HA-Ad) ${ }_{30.5}$ multilayers. Fluorescence images of the assembled 3D structures when the top PDMS layer (f) overlapped with the bottom layer and $(\mathrm{g})$ was tilted at a particular angle; the upper pictures are corresponding schematic illustrations of the 3D structures; note that the PDMS sticks premixed with green fluorescent dyes were modified with (PLL/HA-Ad) 30.5 multilayers while the red ones have $(\mathrm{PLL} / \mathrm{HA}-\mathrm{CD})_{30.5}$ multilayers.

have interconnected pores at the cellular level, indicating the feasibility of forming scaffolds for cell migration and efficient nutrition/waste exchange during metabolic processes. Now the development of MSA is still in its early stages; advanced functions, such as 3D site-selective cell adhesion, have not yet been realized with 3D MSA structures. The challenge lies in creating anisotropic chemical environment that exhibits different cell adhesion properties in 3D structures. In other words, the chemical or biological properties of each building block can be efficiently tailored to induce 3D chemical diversity after directed assembly. Meanwhile, the MSA construction process should be biocompatible with all the materials or chemicals used and the assembly conditions employed low-toxic to cells.

Herein, we have developed a biocompatible MSA strategy that combines $3 \mathrm{D}$ alignment and chiral molecules to fabricate 3D structures with internal chemical diversity and achieved site-selective cell adhesion. The selectivity in cell adhesion was realized by exploiting the opposite chiralities of poly-L-lysine (PLL) - and poly-D-lysine (PDL)-modified polydimethylsiloxane (PDMS) building blocks. Spatial distribution of the two opposing chiral materials and stabilization of the $3 \mathrm{D}$ structures were achieved by (1) magnetic-field-assisted 3D alignment of PDMS and (2) subsequent interfacial binding of host/guest molecular recognition ${ }^{22,23}$ between surface groups of $\beta$ cyclodextrin (CD) and adamantane (Ad) modified on PDMS. To be specific, the layer-by-layer (LbL) assembly technique was used to modify multiple functional groups on PDMS building blocks: ${ }^{24}$ PLL or PDL was used as the polycation and hyaluronic acid (HA) or carboxylated chitosan (CCS) that were partially grafted with $\mathrm{CD}$ or Ad was the polyanion. The induced chiral molecules and host/guest molecular interactions were demonstrated to function orthogonally for their designed purposes. The resulted 3D 
MSA structures showed selective cell adhesion on targeted surfaces within the structure. This principle was proven to be effective to five different cell lines including human lung fibroblast cells (HLFCs), human osteosarcoma cells (MG63), human umbilical vein endothelial cells (HUVECs), rabbit aortic smooth muscle cells (CCC-SMC-1), and PC12 cells. We envision that this facile MSA method can be used to develop a platform that resembles a chemically/biologically diverse $3 \mathrm{D}$ microenvironment for studying cell/material interactions and even tissue formation.

\section{RESULT AND DISCUSSION}

We considered several main factors, viz. cell adhesion, biocompatibility, and interfacial binding of building blocks, for designing and fabricating MSA building blocks. For example, the PLL and PDL molecules are commercially available biomolecules with opposite chiralities ${ }^{25}$ that are known to enhance cell adhesion based on electrostatic interactions between their positive charges with negatively charged ions of the cell membrane. In addition, PDL and PLL, together with the glucose-based HA and CCS molecules, are biocompatible polycations or polyanions bearing positive or negative charges. These molecules can be used to realize electrostatic interaction-driven $\mathrm{LbL}$ modification of building blocks, which makes integrating multiple functional groups on PDMS building blocks possible. We further induced host/ guest molecular interactions by partially substituting carboxylic acid groups on HA and CCS with CD or Ad groups (Figure 1a). When the grafted percentage of CD or Ad groups was low, for example, HA-CD (7.7\%), HA-Ad (3.0\%), and CCS-CD (13.7\%) (Supporting Information (SI) Figure S1), the negative charges of the carboxylic acid groups could be retained for the LbL with PLL or PDL; meanwhile, the CD and Ad groups could be incorporated for later interfacial binding during the MSA process.

The above-mentioned chemical species were used to conduct a stepwise MSA procedure for fabricating a 3D structure (Figure $1 \mathrm{~b}-\mathrm{d}$ ). First of all, the building blocks of PDMS sticks $(2 \mathrm{~mm} \times 150 \mu \mathrm{m} \times 60 \mu \mathrm{m})$ were embedded with $\mathrm{Fe}_{3} \mathrm{O}_{4}$ magnetic nanoparticles (SI Scheme S1) and alternately immersed in polycation/polyanion solutions (e.g., PLL/HACD, PLL/HA-Ad, PDL/CCS-CD solutions) to conduct LbL surface modification (Figure $1 \mathrm{~b}, \mathrm{c}$ ), leading to polyelectrolyte multilayers, such as a (PLL/HA-CD $)_{30}$-PLL multilayer, on PDMS. The number 30 in subscript represents the cyclic number of alternating immersions and an extra single layer of PLL was deposited at outmost. For simplicity, this multilayer has been referred as (PLL/HA-CD) 30.5 . To create diverse building blocks with different surface chemistry, we modified quartz substrates and PDMS building blocks with either (PLL/ HA-CD $)_{30.5}$ or (PLL/HA-Ad $)_{30.5}$ or (PDL/CCS-CD $)_{30.5}$ multilayers, which turned the hydrophobic PDMS into hydrophilic and had a thickness below $150 \mathrm{~nm}$ with a suitable Young's modulus for cell adhesion (SI Figures S2 and S3; Tables S1-S3). Second, we used the magnetic pick-and-place manipulation in water to move the as-prepared PDMS building blocks to designated positions (Figure 1d). Directed manipulation was realized because PDMS building blocks with $\mathrm{Fe}_{3} \mathrm{O}_{4}$ nanoparticles showed fast magnetic-responsive properties upon being exerted with a magnetic field produced by a permanent magnet $(1.0-2.0 \mathrm{mT}$ from the distance of $\sim 1.5 \mathrm{~cm}$; SI Figures S4 and S5). Third, the PDMS building blocks modified with (PLL/HA-Ad) 30.5 were immobilized onto the substrate modified with (PLL/HA-CD) 30.5 by the interfacial molecular interaction of $\mathrm{CD} / \mathrm{Ad}$, during which water was drained off to reduce the water level for achieving close interfacial contact. To quantify the interfacial interactive forces, we determined the contacting-time-dependent binding strength between PDMS building blocks with CD/Ad interactions via an in situ force measurement method. ${ }^{16}$ The measured forces increased rapidly and leveled off at around $325 \mathrm{~N} / \mathrm{m}^{2}$ after $10 \mathrm{~min}$ contact (Figure 1e). It has been previously reported that such strong forces can promote the association of macroscopic building blocks ${ }^{26}$ owing to the multivalent effects at the interface. Finally, the above stepwise magnetic manipulation and interfacial molecular interactions were applied in a cyclic fashion to stack the target PDMS building blocks, leading to $3 \mathrm{D}$ ordered structures of designed geometry and surface chemistry. For example, PDMS sticks premixed with red or green fluorescent dyes and modified with $\mathrm{CD}$ or Ad groups assembled into 3D ordered structures with either the top layer being fully overlapped with the bottom layer (Figure 1f) or being tilted at a particular angle (Figure $1 \mathrm{~g}$ ). The whole MSA process is mild and facile to build 3D ordered structures on demand, indicating its potential for biological uses. Moreover, coupling multiple groups (e.g., chiral molecules and host/guest groups) has enriched the functions of MSA building blocks. This can be attributed to the additive and synergetic features of multiple supramolecular interactions in LbL assembled multilayers. ${ }^{19,27}$

Although we have demonstrated the construction of a 3D ordered structure via the MSA method, the biocompatibility of all the involved materials and the whole process is yet to be evaluated. Therefore, we determined the cell cytotoxicity of the synthetic polyelectrolytes, the LbL-assembled multilayers, and the 3D MSA structure using a standard MTT assay or a live/ dead assay. The results showed that the viability of the cells in the presence of these polyelectrolytes, such as PLL, PDL, HACD, HA-Ad, and CCS-CD, was higher than or comparable to the viability of the cells in the control groups at a concentration lower than $0.2 \mathrm{mg} / \mathrm{mL}$ (Figure 2a-c; SI Figure S6). To evaluate the biocompatibility of the LbL-assembled multilayers, we placed the substrates modified with these multilayers at the bottom of 24-well cell culture microplates and evaluated the cell viability with MTT assays after cell culture for 12, 24, and $48 \mathrm{~h}$ (Figure 2d). The films modified with either (PLL/ HA-CD $)_{30.5}$ or (PLL/HA-Ad) 30.5 multilayers exhibited high cell viability and cell proliferation properties after $48 \mathrm{~h}$ of culture. To further observe the cell morphology, we have applied the live/dead assay to dye the cells for the observation with a fluorescence microscopy. As shown in Figure 2e,f, the HLFCs in the presence of (PLL/HA-CD) 30.5 and (PLL/HAAd) ${ }_{30.5}$ multilayers exhibited high cell coverage and spindle-like morphologies consisting of fibroblast cells, indicating good cell compatibility. Taken together, either the above individual chemicals or thin films fabricated via LbL assembly of these polyelectrolytes have been proven with a low cell toxicity.

Based on the above low-toxic chemicals and multilayers, we further clarify whether the 3D MSA structures with porous structures built from these chemical species could facilitate cell adhesion. Therefore, we conducted the stepwise magnetic pick-and-place and interfacial bindings of PDMS building blocks $(2 \mathrm{~mm} \times 150 \mu \mathrm{m} \times 60 \mu \mathrm{m})$ modified with either (PLL/ HA-CD $)_{30.5}$ or (PLL/HA-Ad) ${ }_{30.5}$ multilayers following the procedure in Figure 1d. We obtained a three-layer stacked structure with a well-defined porous structure on a quartz 

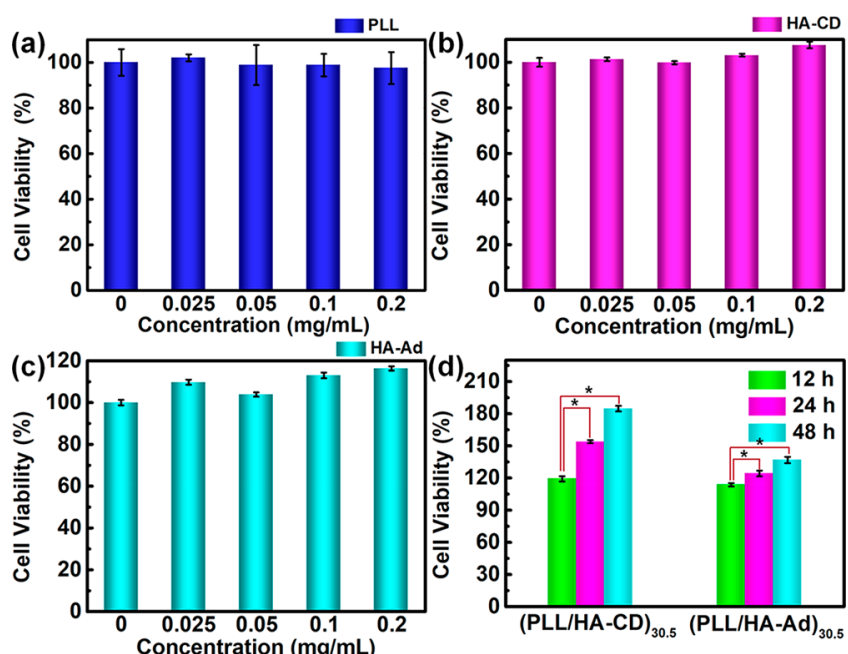

(e)
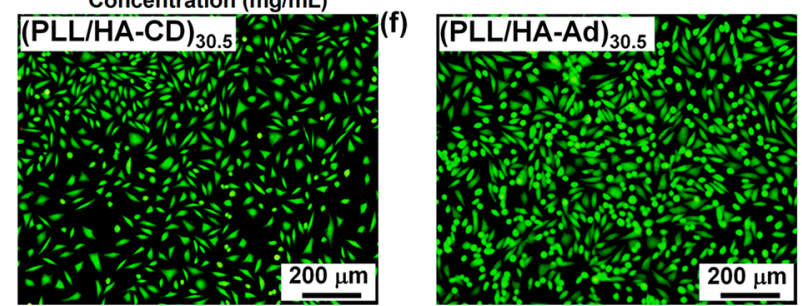

Figure 2. Cell viability studied using (a) PLL, (b) HA-CD, (c) HAAd, (d) (PLL/HA-CD) 30.5 , and (PLL/HA-Ad) 30.5 multilayers. Fluorescence images of HLFCs on flat PDMS sheets modified with (e) (PLL/HA-CD $)_{30.5}$ and (f) (PLL/HA-Ad) $)_{30.5}$ multilayers.

substrate modified with a (PLL/HA-CD $)_{30.5}$ multilayer: the second layer is perpendicularly aligned relative with the first layer and the third layer is tilted by 45 deg relative with the second layer; in each layer, all PDMS building blocks were aligned parallelly with a uniform space between each other (Figure $3 \mathrm{a}-\mathrm{f}$ ). When HLFCs were seeded onto the above 3D ordered structures of PDMS, the cells exhibited increased coverage with the increasing cell culture time from 12 to $48 \mathrm{~h}$; the local magnification displays spindle-like morphologies of healthy fibroblast cells (Figure 3b,d,f). Thus, the biocompatible nature of all the synthetic chemicals used and the MSA procedure was confirmed.

Because the above 3D MSA structures consist of similar surface chemistry of the (PLL/HA-CD or Ad) 30.5 multilayers, we could observe high cell density throughout the $3 \mathrm{D}$ structure and the substrates, namely nonselective cell adhesion (Figure 3e). Normally, this phenomenon is inevitable due to common cell seeding techniques simply by gravity settling without any selective adhesion mechanism. There remains a question whether it is possible to achieve site-selective cell adhesion within the established 3D microenvironment. Creating chemical diversity in the structure and meanwhile ensure biocompatibility should be a feasible solution. Hence, we considered the most distinctive biochemical signature of molecular chirality that is widely observed in life to influence many biological events. ${ }^{28-30}$ Several reports state that lefthanded molecules are preferred for cell adhesion over righthanded molecules. ${ }^{8,10}$ We have chosen commercially available PLL and PDL molecules as chiral systems to direct cell adhesion and build 3D MSA structures using the molecular recognition systems consisting of HA-CD, HA-Ad, and CCSCD.
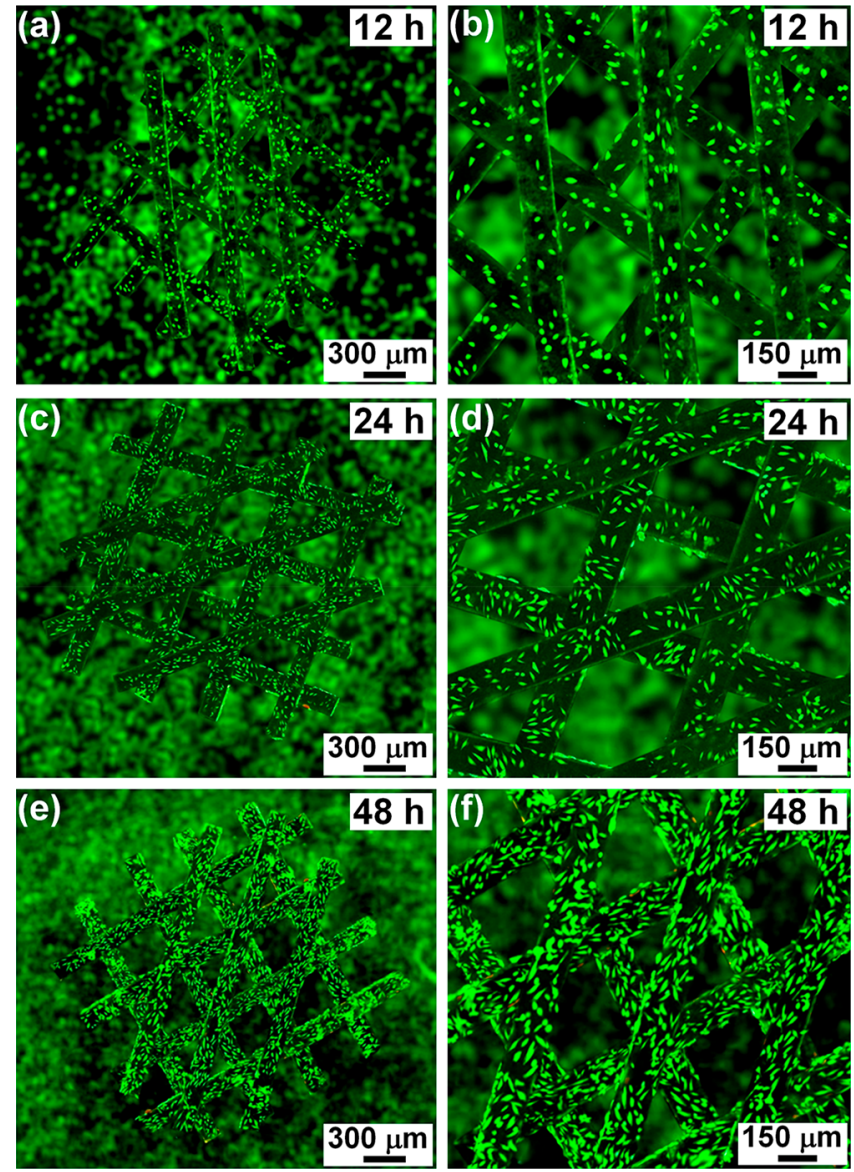

Figure 3. Fluorescence images of HLFCs on 3D ordered structures made of PDMS modified with (PLL/HA-CD) 30.5 or (PLL/HAAd) $)_{30.5}$ multilayers after cell culture for (a,b) 12, (c,d) 24, and (e,f) 48 h. $(b, d, f)$ are corresponding magnified images of $(a, c, e)$. The quartz substrates supporting the $3 \mathrm{D}$ structures were modified with (PLL/ HA-CD) 30.5 multilayers.

The chirality of the polyelectrolyte multilayers after incorporating chiral molecules of PLL and PDL, that is, $(\mathrm{PLL} / \mathrm{HA}-\mathrm{CD})_{30.5}$ and (PDL/HA-CD) $)_{30.5}$ multilayers, was determined using a probe molecule of tetrasodium-mesotetra(4-sulfonatophenyl)porphine dodecahydrate (TPPS) following a reported procedure. ${ }^{25}$ The multilayers were characterized using circular dichroism spectroscopy (Figure 4a; SI Figure S7) and exhibited opposite signals of complementary shapes, indicating that the opposite chiralities of PLL or PDL were retained in the modified multilayers and could direct different cell binding with such distinctive chemical properties. We confirmed this hypothesis by comparing cell adhesion on substrates that are modified with the (PLL/HA-CD $)_{30.5}$ multilayer and the (PDL/CCS-CD) 30.5 multilayer. After applying live/dead assays, the cell density on the (PLL/HA-CD) 30.5 multilayer (Figure $4 \mathrm{~b}$ ) is much higher than that on the (PDL/CCS-CD) 30.5 multilayer (Figure 4c). The experiments of this cell adhesion phenomenon have been repeated 20 times and in $68.4 \%$ of them we observed a better cell adhesion on multilayers containing PLL than that on the PDL multilayers (26.3\% display comparable effects and only $5.3 \%$ show the opposite). In addition to HLFCs, another four kinds of cell lines including MG63 cells, HUVECs, CCCSMC-1 cells, and PC12 cells, also exhibited similar selective adhesion properties, which can be attributed to the observed 

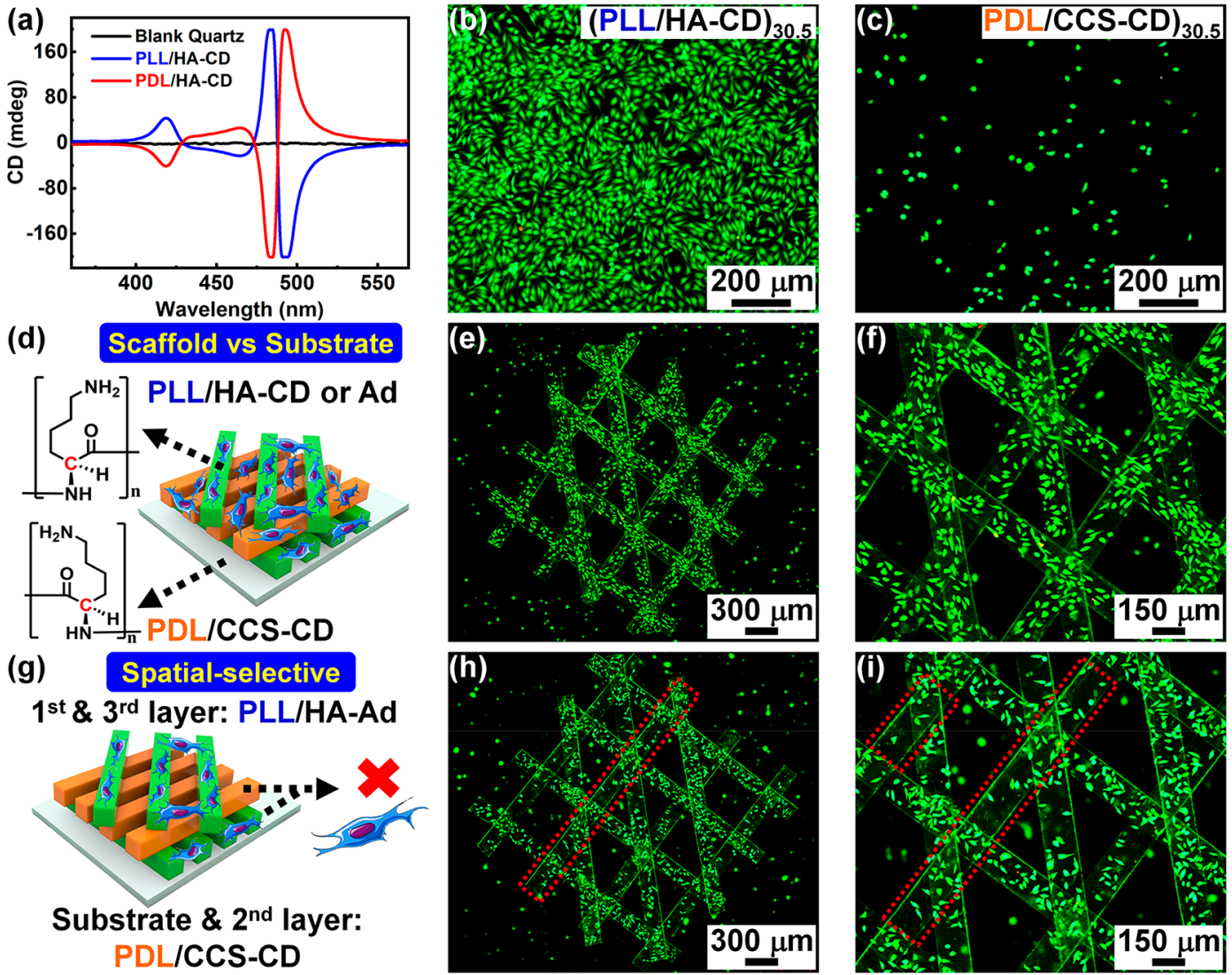

Figure 4. (a) Circular dichroism (CD) spectra of (PLL/HA-CD) 30.5 and (PDL/HA-CD) $)_{30.5}$ multilayers. Fluorescence images after applying live/ dead assays to PDMS modified with (b) (PLL/HA-CD) 30.5 and (c) (PDL/CCS-CD) 30.5 multilayers (cell culture time: $48 \mathrm{~h}$ ). Schematic illustrations and fluorescence images for 3D MSA structures subjected to live/dead assays (full image and local magnification): (d-f) The scaffold is modified with (PLL/HA-CD or Ad) $)_{30.5}$ multilayers while the substrate is modified with (PDL/CCS-CD) 30.5 multilayer; $(\mathrm{g}-\mathrm{i}$ ) the first and third layer of PDMS are modified with the (PLL/HA-Ad) $)_{30.5}$ multilayer while the second layer (highlighted in the red dashed boxes) and the substrate are modified with the $(\mathrm{PDL} / \mathrm{CCS}-\mathrm{CD})_{30.5}$ multilayer.

chirality difference (SI Figures S8-S11). The different cell adhesion on $2 \mathrm{D}$ films means the possibility to induce $3 \mathrm{D}$ selective adhesion.

To verify whether the differentiating mechanism of $2 \mathrm{D}$ films based on molecular chirality could direct 3D site-selective adhesion. We started with selective cell adhesion between the $3 \mathrm{D}$ scaffold and the substrate, that is, the cells should preferably adhere onto the $3 \mathrm{D}$ structure rather than the substrate, which is a challenge in the conventional gravitydominant cell seeding technique. As illustrated in Figure 4d, the substrate was modified using a low-cell-adhesive (PDL/ CCS-CD $)_{30.5}$ multilayer and PDMS building blocks with highcell-adhesive multilayers of (PLL/HA-CD) 30.5 or (PLL/HAAd) $)_{30.5}$. The obtained 3D MSA structure with differentiating chemistry after cell culture (HLFCs) for $48 \mathrm{~h}$ and live/dead staining (Figure 4e,f) shows totally different results when comparing with the 3D structure of identical chemistry (Figure $3 \mathrm{e}, \mathrm{f})$ : most cells attached well onto the $3 \mathrm{D}$ scaffold and did not adhere well onto the quartz substrate. While the cells did not adhere well to the substrate, the cell density on the 3D scaffold remained high, indicating that the $(\mathrm{PDL} / \mathrm{CCS}-\mathrm{CD})_{30.5}$ multilayer modified on the substrate does not damage the biocompatibility of the system or inhibit cell growth on the upper scaffold. The next challenge is to create different surface chemistry within the 3D MSA structures and realize siteselective cell adhesion. As designed in Figure 4g, the internal chemical diversity was realized by modifying the substrate and the second PDMS layer using the low-cell-affinitive (PDL/ CCS-CD $)_{30.5}$ multilayer while other PDMS layers were modified using multilayers containing (PLL/HA-Ad) 30.5 multilayers. Under identical conditions of cell culture of HLFCs for $48 \mathrm{~h}$ and applying live/dead assays, we observed a much lower cell density on the second layer and the substrate compared to the cell density on the other layers (Figure 4h,i), leading to the cell preference of surfaces with PLL over those with PDL. Thus, by simply using the opposite chirality without changing the main components in the polyelectrolyte multilayers, we achieved site-selective cell adhesion on demand in 3D structures based on the fabrication method of MSA.

\section{CONCLUSION}

We have developed a biocompatible MSA method to fabricate $3 \mathrm{D}$ ordered structures exhibiting the property of $3 \mathrm{D}$ siteselective cell adhesion at designated positions. A biocompatible microenvironment and internal chemical diversity are the prerequisites. We address the issue of improving the biocompatibility by using the homopolypeptides of PLL and PDL that are widely applied in cell culture, polysaccharides (CCS and HA), a cyclic oligosaccharide (CD), and a molecule for common biomedical uses (Ad derivatives). The surface chemistry difference in $3 \mathrm{D}$ structures were realized based on the facile assembly of PDMS building blocks modified with different LbL polyelectrolyte multilayers. Further selective cell affinity was achieved in $3 \mathrm{D}$ structures using polyelectrolyte 
multilayers of opposite chiralities: the PDMS modified with $(\text { PLL/HA-CD })_{30.5}$ or (PLL/HA-Ad) $)_{30.5}$ multilayers exhibited significantly higher cell density than the area modified with a (PDL/CCS-CD $)_{30.5}$ multilayer. This strategy has been proven effective to five different cell lines including HLFCs, MG63 cells, HUVECs, CCC-SMC-1 cells, and PC12 cells. We envision that this MSA method can present a build-on-demand concept to fabricate a complex 3D scaffold with chemical and biological diversity, such as controlled 3D distribution of specific biomolecules, different cell lines etc.

\section{EXPERIMENTAL SECTION}

Materials. The following chemicals were used as purchased. Hyaluronic acid sodium salt from Streptococcus equi was from SigmaAldrich. $N$-(3-(dimethylamino)propyl)- $N^{\prime}$-ethylcarbodiimide hydrochloride (EDC) and $\mathrm{N}$-hydroxysuccinimide (NHS) were from J\&K. 1-Adamantanamine (Ad) was from Alfa Aesar. 6-Amino- $\beta$-cyclodextrin was from Shandong Binzhou Zhiyuan Biotechnology. Calcein$\mathrm{AM}$, propidium iodide, and carboxylated chitosan were from Aladdin. PDMS (Sylgand 184) was from Dow Corning. $\mathrm{Fe}_{3} \mathrm{O}_{4}$ magnetic nanoparticles (MNPs) in the form of dry powder was from Beijing DK Nanotechnology. TPPS was from Strem Chemicals. PLL and PDL were from Shanghai Yuanye Biotechnology. Other normal reagents such as anhydrous dimethyl sulfoxide, citrate-phosphate buffer, and $75 \%$ ethanol (ethanol: water $=75 \mathrm{vt} \%$ ) were from Sinopharm Chemical Reagent Beijing. Consumables for cell culture such as 96-well and 24-well cell culture microplates, cell culture flasks (T-25 polystyrene flasks), and pipet tips were from Corning Incorporated.

HLFCs and L929 cells were generously provided by Institute of Basic Medicine Science, College of Life Science, Chinese PLA General Hospital. HUVECs, CCC-SMC-1, MG63 cells, and PC12 cells were purchased from Procell Biotechnology. Cell culture materials: minimum Eagle's medium (MEM), high-glucose Dulbecco's modified Eagle's medium (DMEM), Roswell Park Memorial Institute medium (RPMI-1640), penicillin-streptomycin solutions, and trypsin $0.25 \%$ solutions from Hyclone; fetal bovine serum (FBS, 10 wt \%) from BBI Life Sciences; thiazolyl blue tetrazolium bromide (MTT) from Solaribio Life Sciences; recombinant human insulin and nonessential amino acids from Procell Biotechnology.

Instruments. The ${ }^{1} \mathrm{H}$ NMR spectra of the products were obtained with a Bruker AV400 NMR spectrometer at $400 \mathrm{MHz}$. The PDMS building blocks were cut with a freezing microtome (CM1950, Leica). The interfacial binding forces were measured with a Dynamic Contact Angle Measuring Device and Tensionmeter (DCAT21, Dataphysics, Germany). The circular dichroism spectra were measured with a circular dichroism spectrometer (J-810, Jasco). MTT assay was evaluated with a microplate reader (Multiskan FC, Thermo Fisher Scientific) at $570 \mathrm{~nm}$. The fluorescence images of cells were taken with a fluorescence microscopy (XSP-63B, Shanghai Optical Instrument Factory). We used a permanent magnet $(5 \mathrm{~cm} \times 1 \mathrm{~cm} \times 1 \mathrm{~cm})$ for the magnetic manipulation of pick-and-place; the distance between the magnet and building blocks were kept at about $1.5 \mathrm{~cm}$, which led to a magnetic field strength of $1-2 \mathrm{mT}$.

Synthesis of HA-CD. Hyaluronic acid sodium salt $(2.17 \mathrm{mmol}, 100$ $\mathrm{mg}$ ) was dissolved in $20 \mathrm{~mL}$ PBS buffer solution (0.2 M, pH 7.2). EDC $(0.87 \mathrm{mmol}, 168 \mathrm{mg})$ and NHS $(0.87 \mathrm{mmol}, 100 \mathrm{mg})$ were dissolved in $5 \mathrm{~mL}$ PBS buffer solution and then dropped into the HA solution. The mixture was stirred for $30 \mathrm{~min}$ at room temperature. Subsequently, 6-amino- $\beta$-cyclodextrin $(0.44 \mathrm{mmol}, 500 \mathrm{mg})$ dissolved in $20 \mathrm{~mL}$ PBS solution was dropped into the above mixture, followed by stirring for $24 \mathrm{~h}$ at room temperature. Finally, the reacted mixture was dialyzed for 5 days in a dialytic bag (molecular weight cut off $(M W C O)=3500)$ and frozen dried. The ${ }^{1} \mathrm{H}$ NMR spectrum of the HA-CD in $\mathrm{D}_{2} \mathrm{O}$ is shown in SI Figure S1a.

Synthesis of HA-Ad. Hyaluronic acid sodium salt $(2.17 \mathrm{mmol}, 100$ $\mathrm{mg}$ ) was dissolved in $20 \mathrm{~mL}$ PBS buffer solution (0.2 M, pH 7.2). EDC $(0.87 \mathrm{mmol}, 168 \mathrm{mg})$ and NHS $(0.87 \mathrm{mmol}, 100 \mathrm{mg})$ were dissolved in $5 \mathrm{~mL}$ PBS buffer solution and then dropped into the HA solution. The mixture was stirred for $30 \mathrm{~min}$ at room temperature. Then 1-adamantanamine $(0.33 \mathrm{mmol}, 50 \mathrm{mg})$ dissolved in $20 \mathrm{~mL}$ PBS solution was dropped into the above mixture, followed by stirring for $24 \mathrm{~h}$ at room temperature. The reacted mixture was dialyzed for 5 days in a dialytic bag $($ MWCO $=3500)$ and frozen dried. The ${ }^{1} \mathrm{H}$ NMR spectrum of HA-Ad in $\mathrm{D}_{2} \mathrm{O}$ is displayed in SI Figure S1b.

Synthesis of CCS-CD. Carboxylated chitosan (179 mg), EDC (1.00 $\mathrm{mmol}, 192 \mathrm{mg})$, and NHS $(1.00 \mathrm{mmol}, 115 \mathrm{mg}$ ) were dissolved in 40 $\mathrm{mL}$ PBS (0.2 M, pH 7.4) and stirred for $30 \mathrm{~min}$ at room temperature. Then 6-amino- $\beta$-cyclodextrin $(1.00 \mathrm{mmol}, 1135 \mathrm{mg})$ dissolved in 20 $\mathrm{mL}$ PBS solution was dropped into the above mixture, followed by stirring for $8 \mathrm{~h}$ at room temperature. The reacted mixture was dialyzed for 5 days $(\mathrm{MWCO}=3500)$ and freeze-dried. The ${ }^{1} \mathrm{H}$ NMR spectrum of CCS-CD in $\mathrm{D}_{2} \mathrm{O}$ is shown in SI Figure S1c.

Fabrication of PDMS Building Blocks. As schematically illustrated in SI Scheme S1, PDMS prepolymer $(10 \mathrm{~g})$, its curing agent $(1 \mathrm{~g})$ and $\mathrm{Fe}_{3} \mathrm{O}_{4}$ magnetic nanoparticles $(100 \mathrm{mg})$ were mixed, degassed and sandwiched between two glass slides spaced by one or two $150-\mu \mathrm{m}$ thick cover glasses at two ends. After heating at $65{ }^{\circ} \mathrm{C}$ for $4 \mathrm{~h}$, the cured PDMS thin films were further manufactured according to different purposes: (1) For MTT evaluation, 2D PDMS films (300$\mu \mathrm{m}$-thick) with a size fitting the wells of the cell culture microplates were cut with a hole puncher; (2) for PDMS stick building blocks, the thin PDMS films $(150-\mu$ m-thick) were cut with a knife into a dimension of $2 \mathrm{~mm} \times 2 \mathrm{~mm} \times 150 \mu \mathrm{m}$, embedded in a resin (TissueTek O.C.T. Compound) to be frozen, and cut into the final dimension of $2 \mathrm{~mm} \times 150 \mu \mathrm{m} \times 60 \mu \mathrm{m}$ with a freezing microtome.

Surface Modification of PDMS Building Blocks or Quartz Substrates. All surface modification was conducted with a layer-bylayer assembled technique. Taking the modification of the (PLL/HACD) 30.5 multilayer on PDMS sticks as an example, the cleaned PDMS was treated by plasma to result in a hydrophilic surface and then immersed in PLL (aq, $0.5 \mathrm{mg} / \mathrm{mL}$ ) for $12 \mathrm{~h}$ as the first layer. Later, the PDMS was alternately immersed in HA-CD (aq, $1 \mathrm{mg} / \mathrm{mL})$ for 5 $\min$ and PLL (aq, $0.5 \mathrm{mg} / \mathrm{mL}$ ) for $5 \mathrm{~min}$; between each immersion step, the PDMS was cleaned with deionized water to remove excessive physical adsorption. The above alternate immersion was repeated until the wanted number of layers was reached, for example, (PLL/ HA-CD $)_{30.5}$, (PLL/HA-Ad) 30.5 , and (PDL/CCS-CD) $)_{30.5}$.

Circular Dichroism of Chiral Species. We checked the chirality of PLL and PDL solutions with a commonly used probe molecule of TPPS. The TPPS solution $(20 \mu \mathrm{M}$ in a $\mathrm{pH} 3.0$ citrate-phosphate buffer) was mixed with a PLL or a PDL solution $(100 \mu \mathrm{M}$ in a $\mathrm{pH} 3.0$ citrate-phosphate buffer) at an equal volume by stirring at room temperature for $2 \mathrm{~h}$ to induce the aggregation of TPPS. ${ }^{25}$ The quartz substrates modified with (PLL/HA-CD $)_{30.5}$, (PDL/HA-CD $)_{30.5}$, and $(\mathrm{PDL} / \mathrm{CCS}-\mathrm{CD})_{30.5}$ multilayers were immersed in a TPPS solution (10 $\mu \mathrm{M}$ in a $\mathrm{pH} 3.0$ citrate-phosphate buffer) for $30 \mathrm{~min}$. After rinsing with deionized water, circular dichroism spectra of the quartz substrates modified with (PLL/HA-CD $)_{30.5}$, or (PDL/HA-CD $)_{30.5}$ multilayers were measured and shown in Figure $4 \mathrm{a}$. The circular dichroism spectrum of the (PLL/CCS-CD $)_{30.5}$ or (PDL/CCS-CD $)_{30.5}$ multilayer was obtained by measuring the TPPS solutions after immersing the quartz substrate modified with the (PLL/CCS-CD) 30.5 or (PDL/CCS-CD $)_{30.5}$ multilayer into the TPPS solutions for $30 \mathrm{~min}$ (SI Figure S7).

Preparation of the Medium for Cell Culture. The medium for the culture of HLFCs consists of DMEM, FBS, and penicillin/ streptomycin $(\mathrm{P} / \mathrm{S})$ with a volume ratio of 100:10:1. The medium for the culture of HUVECs contains the mixture of DMEM, FBS and $\mathrm{P} / \mathrm{S}$ (volume ratio $=100: 10: 1)$, recombinant human insulin $(0.01$ $\mathrm{mg} / \mathrm{mL}$ ) and nonessential amino acids (1 vt \%). The medium for the culture of CCC-SMC-1 has the mixture of DMEM, FBS and P/S with a volume ratio of 100:15:1. The medium for the culture of PC12 and L929 cells contains the mixture of RPMI-1640, FBS and P/S (volume ratio $=100: 10: 1)$. The special medium of MG63 cells consists of the mixture of MEM, FBS and P/S (volume ratio = 100:10:1). The cell culture condition was set as $5 \% \mathrm{CO}_{2}$ at $37{ }^{\circ} \mathrm{C}$. 
MTT of Chemicals. The cell viability was evaluated with a standard MTT assay. Cells (e.g., HLFCs) were seeded into a 96-well microplate with a density of $1 \times 10^{4}$ cells per well and cultured for $24 \mathrm{~h}$. Then, we added a solution of one certain chemical, e.g. PLL, PDL, HA-Ad, HA-CD or CCS-CD with varied concentrations of $0.025,0.05,0.1$, and $0.2 \mathrm{mg} / \mathrm{mL}$. After culture for another $24 \mathrm{~h}$ in $5 \%$ $\mathrm{CO}_{2}$ at $37^{\circ} \mathrm{C}$ for $24 \mathrm{~h}$, we added MTT $(5 \mathrm{mg} / \mathrm{mL}$, in PBS) solutions to each well and kept for $4 \mathrm{~h}$. The 96-well microplates were then measured via a microplate reader at $570 \mathrm{~nm}$. The cell viability was calculated based on the absorbance ratio of the wells added with chemicals and the wells as controls (Figure $2 \mathrm{a}-\mathrm{c}$ and Figure S6).

MTT of Multilayers. Flat PDMS substrates modified with (PLL/ HA-CD $)_{30.5}$ or (PLL/HA-Ad) $)_{30.5}$ multilayers were placed at the bottom of 24-well microplates and sterilized by immersing in $75 \%$ ethanol under UV light for $30 \mathrm{~min}$, followed by cleaning with PBS buffer ( $\mathrm{pH} 7.4)$ for three times. L929 cells were seeded with a density of $1 \times 10^{5}$ cells per well and cultured for 12,24 , and $48 \mathrm{~h}$. We added MTT solutions $(5 \mathrm{mg} / \mathrm{mL}$, in PBS) to each well and kept for $4 \mathrm{~h}$. The solutions were then measured via a microplate reader at $570 \mathrm{~nm}$. The cell viability was calculated based on the absorbance ratio of the wells with and without PDMS films.

Selective Cell Adhesion Directed by Chiral Multilayers. The observation of cell adhesion on $2 \mathrm{D}$ multilayers or $3 \mathrm{D}$ ordered structures in Figures 2-4 and SI Figures S8-11 include the following primary steps. For example, (1) a quartz substrate modified with the $(\mathrm{PLL} / \mathrm{HA}-\mathrm{CD})_{30.5}$ multilayer was placed in a 24-well microplates and sterilized in $75 \%$ ethanol under UV light for $30 \mathrm{~min}$, followed by cleaning with a PBS buffer ( $\mathrm{pH}$ 7.4) for three times. (2) After immersing in the culture medium for $12 \mathrm{~h}$, cells were seeded and cultured in corresponding mediums in $5 \% \mathrm{CO}_{2}$ at $37^{\circ} \mathrm{C}$ for $12 \mathrm{~h}, 24$ $\mathrm{h}$, and $48 \mathrm{~h}$. (3) At each time interval, live/dead assays were used to dye the cells for the observation of the cell adhesion with a fluorescence microscopy; we used the excitation wavelengths of 494 and $528 \mathrm{~nm}$.

\section{ASSOCIATED CONTENT}

\section{(5) Supporting Information}

The Supporting Information is available free of charge at https://pubs.acs.org/doi/10.1021/acsami.1c05181.

${ }^{1} \mathrm{H}$ NMR spectra of HA-CD, HA-Ad and CCS-CD; wettability, thickness, and Young's modulus of LbL assembled multilayers; morphology and magnetic properties of $\mathrm{Fe}_{3} \mathrm{O}_{4}$ nanoparticles and the PDMS$\mathrm{Fe}_{3} \mathrm{O}_{4}$ building blocks; MTT results of PDL and CCS$\mathrm{CD}$; circular dichroism spectra of TPPS, PLL, PDL, (PLL/CCS-CD $)_{30.5}$ multilayers, and (PDL/CCS$\mathrm{CD})_{30.5}$ multilayers; fluorescence images of cell adhesion (MG63, HUVECs, CCC-SMC-1, and PC12 cells) (PDF)

\section{AUTHOR INFORMATION}

\section{Corresponding Authors}

Mengjiao Cheng - State Key Laboratory of Chemical Resource Engineering, Beijing Laboratory of Biomedical Materials, Beijing Advanced Innovation Center for Soft Matter Science and Engineering, Beijing University of Chemical Technology, Beijing 100029, China; (1) orcid.org/ 0000-0002-1137-3545; Email: chengmj@mail.buct.edu.cn

Feng Shi - State Key Laboratory of Chemical Resource Engineering, Beijing Laboratory of Biomedical Materials, Beijing Advanced Innovation Center for Soft Matter Science and Engineering, Beijing University of Chemical Technology, Beijing 100029, China; (i) orcid.org/0000-0001-5897-

5116; Email: shi@mail.buct.edu.cn

\section{Authors}

Changyu Wang - State Key Laboratory of Chemical Resource Engineering, Beijing Laboratory of Biomedical Materials, Beijing Advanced Innovation Center for Soft Matter Science and Engineering, Beijing University of Chemical Technology, Beijing 100029, China; 이이이.org/0000-0001-8869-1229

Cuiling Lin - State Key Laboratory of Chemical Resource Engineering, Beijing Laboratory of Biomedical Materials, Beijing Advanced Innovation Center for Soft Matter Science and Engineering, Beijing University of Chemical Technology, Beijing 100029, China

Rui Ming - State Key Laboratory of Chemical Resource Engineering, Beijing Laboratory of Biomedical Materials, Beijing Advanced Innovation Center for Soft Matter Science and Engineering, Beijing University of Chemical Technology, Beijing 100029, China

Xiangxin Li - State Key Laboratory of Chemical Resource Engineering, Beijing Laboratory of Biomedical Materials, Beijing Advanced Innovation Center for Soft Matter Science and Engineering, Beijing University of Chemical Technology, Beijing 100029, China

Pascal Jonkheijm - Department of Molecules and Materials, Faculty of Science and Technology, MESA+ Institute for Nanotechnology and TechMed Centre, University of Twente, 7500 AE Enschede, The Netherlands; 10 orcid.org/00000001-6271-0049

Complete contact information is available at:

https://pubs.acs.org/10.1021/acsami.1c05181

\section{Author Contributions}

The manuscript was written through contributions of all authors. All authors have given approval to the final version of the manuscript.

\section{Author Contributions}

${ }^{\S}$ C.W. and C.L. contributed equally to this work.

\section{Notes}

The authors declare no competing financial interest.

\section{ACKNOWLEDGMENTS}

This work was supported by the National Science Foundation for Distinguished Young Scholars (51925301), the National Natural Science Foundation of China (21972008), the Science Fund for Distinguished Young Scholars of Beijing Natural Science Foundation (JQ180003), Wanren Plan (wrjh201903), Beijing Nova Program (Z201100006820021), the Fundamental Research Funds for the Central Universities (XK1902).

\section{REFERENCES}

(1) Ovsianikov, A.; Khademhosseini, A.; Mironov, V. The Synergy of Scaffold-Based and Scaffold-Free Tissue Engineering Strategies. Trends Biotechnol. 2018, 36, 348-357.

(2) Mitrousis, N.; Fokina, A.; Shoichet, M. S. Biomaterials for Cell Transplantation. Nat. Rev. Mater. 2018, 3, 441-456.

(3) Kang, H.-W.; Lee, S. J.; Ko, I. K.; Kengla, C.; Yoo, J. J.; Atala, A. A 3D Bioprinting System to Produce Human-Scale Tissue Constructs with Structural Integrity. Nat. Biotechnol. 2016, 34, 312-319.

(4) Visser, C. W.; Kamperman, T.; Karbaat, L. P.; Lohse, D.; Karperien, M. In-Air Microfluidics Enables Rapid Fabrication of Emulsions, Suspensions, and 3D Modular (Bio)Materials. Sci. Adv. 2018, 4, eaao 1175 .

(5) Nikolaev, M.; Mitrofanova, O.; Broguiere, N.; Geraldo, S.; Dutta, D.; Tabata, Y.; Elci, B.; Brandenberg, N.; Kolotuev, I.; Gjorevski, N.; Clevers, H.; Lutolf, M. P. Homeostatic Mini-Intestines through 
Scaffold-Guided Organoid Morphogenesis. Nature 2020, 585, 574578.

(6) Venturini, V.; Pezzano, F.; Català Castro, F.; Häkkinen, H.-M.; Jiménez-Delgado, S.; Colomer-Rosell, M.; Marro, M.; Tolosa-Ramon, Q.; Paz-López, S.; Valverde, M. A.; Weghuber, J.; Loza-Alvarez, P.; Krieg, M.; Wieser, S.; Ruprecht, V. The Nucleus Measures Shape Changes for Cellular Proprioception to Control Dynamic Cell Behavior. Science 2020, 370, eaba2644.

(7) Tam, R. Y.; Yockell-Lelièvre, J.; Smith, L. J.; Julian, L. M.; Baker, A. E. G.; Choey, C.; Hasim, M. S.; Dimitroulakos, J.; Stanford, W. L.; Shoichet, M. S. Rationally Designed 3D Hydrogels Model Invasive Lung Diseases Enabling High-Content Drug Screening. Adv. Mater. 2019, 31, 1806214.

(8) Sun, T.; Han, D.; Rhemann, K.; Chi, L.; Fuchs, H. Stereospecific Interaction between Immune Cells and Chiral Surfaces. J. Am. Chem. Soc. 2007, 129, 1496-1497.

(9) Yao, X.; Hu, Y.; Cao, B.; Peng, R.; Ding, J. Effects of Surface Molecular Chirality on Adhesion and Differentiation of Stem Cells. Biomaterials 2013, 34, 9001-9009.

(10) Zhang, S.; Zhao, G.; Wang, J.; Xie, C.; Liang, W.; Chen, K.; Wen, Y.; Li, X. Organic Solvent-Free Preparation of Chitosan Nanofibers with High Specific Surface Charge and their Application in Biomaterials. ACS Appl. Mater. Interfaces 2021, 13, 12347-12358.

(11) Yu, S.; Gao, Y.; Mei, X.; Ren, T.; Liang, S.; Mao, Z.; Gao, C. Preparation of an Arg-Glu-Asp-Val Peptide Density Gradient on Hyaluronic Acid-Coated poly ( $\varepsilon$-Caprolactone) Film and its Influence on the Selective Adhesion and Directional Migration of Endothelial Cells. ACS Appl. Mater. Interfaces 2016, 8, 29280-29288.

(12) Dou, X.; Wu, B.; Liu, J.; Zhao, C.; Qin, M.; Wang, Z.; Schönherr, H.; Feng, C. Effect of Chirality on Cell Spreading and Differentiation: From Chiral Molecules to Chiral Self-Assembly. ACS Appl. Mater. Interfaces 2019, 11, 38568-38577.

(13) Wei, Y.; Jiang, S.; Si, M.; Zhang, X.; Liu, J.; Wang, Z.; Cao, C.; Huang, J.; Huang, H.; Chen, L.; Wang, S.; Feng, C.; Deng, X.; Jiang, L. Chirality Controls Mesenchymal Stem Cell Lineage Diversification through Mechanoresponses. Adv. Mater. 2019, 31, 1900582.

(14) Harada, A.; Kobayashi, R.; Takashima, Y.; Hashidzume, A.; Yamaguchi, H. Macroscopic Self-Assembly through Molecular Recognition. Nat. Chem. 2011, 3, 34-37.

(15) Cheng, M.; Gao, H.; Zhang, Y.; Tremel, W.; Chen, J.-F.; Shi, F.; Knoll, W. Combining Magnetic Field Induced Locomotion and Supramolecular Interaction to Micromanipulate Glass Fibers: Toward Assembly of Complex Structures at Mesoscale. Langmuir 2011, 27, 6559-6564.

(16) Cheng, M.; Shi, F.; Li, J.; Lin, Z.; Jiang, C.; Xiao, M.; Zhang, L.; Yang, W.; Nishi, T. Macroscopic Supramolecular Assembly of Rigid Building Blocks through a Flexible Spacing Coating. Adv. Mater. 2014, 26, 3009-3013.

(17) Cheng, M.; Shi, F. Precise Macroscopic Supramolecular Assemblies: Strategies and Applications. Chem. - Eur. J. 2020, 26, 15763-15778.

(18) Sinawang, G.; Osaki, M.; Takashima, Y.; Yamaguchi, H.; Harada, A. Biofunctional Hydrogels Based on Host-Guest Interactions. Polym. J. 2020, 52, 839-859.

(19) Cheng, M.; Wang, Y.; Yu, L.; Su, H.; Han, W.; Lin, Z.; Li, J.; Hao, H.; Tong, C.; Li, X.; Shi, F. Macroscopic Supramolecular Assembly to Fabricate 3D Ordered Structures: Towards Potential Tissue Scaffolds with Targeted Modification. Adv. Funct. Mater. 2015, 25, 6851-6857.

(20) Zhang, Q.; Sun, Y.; He, C.; Shi, F.; Cheng, M. Fabrication of 3D Ordered Structures with Multiple Materials via Macroscopic Supramolecular Assembly. Adv. Sci. 2020, 7, 2002025.

(21) Ji, X.; Li, Z.; Liu, X.; Peng, H.-Q.; Song, F.; Qi, J.; Lam, J. W. Y.; Long, L.; Sessler, J. L.; Tang, B. Z. A Functioning Macroscopic "Rubik's Cube" Assembled via Controllable Dynamic Covalent Interactions. Adv. Mater. 2019, 31, 1902365.

(22) Cheng, M.; Zhang, D.; Zhang, S.; Wang, Z.; Shi, F. Tackling the Short-Lived Marangoni Motion Using a Supramolecular Strategy. CCS Chem. 2019, 1, 148-155.
(23) Zhan, S.; Wei, T.; Yu, Q.; Chen, H. Fabrication of Supramolecular Bioactive Surfaces via $\beta$-Cyclodextrin-Based HostGuest Interactions. ACS Appl. Mater. Interfaces 2018, 10, 3658536601.

(24) An, Q.; Huang, T.; Shi, F. Covalent Layer-by-Layer Films: Chemistry, Design, and Multidisciplinary Applications. Chem. Soc. Rev. 2018, 47, 5061-5098.

(25) Koti, A. S. R.; Periasamy, N. Self-Assembly of TemplateDirected J-Aggregates of Porphyrin. Chem. Mater. 2003, 15, 369-371.

(26) Liu, C.; Zhang, Q.; Zhang, Y.; Cheng, M.; Shi, F. Macroscopic Supramolecular Assembly through Adjusting the Surface-Flexibility of the Building Block. Kexue Tongbao 2018, 63, 3650-3657.

(27) Helmers, I.; Niehues, M.; Kartha, K. K.; Ravoo, B. J.; Fernández, G. Synergistic Repulsive Interactions Trigger Pathway Complexity. Chem. Commun. 2020, 56, 8944-8947.

(28) Gim, S.; Fittolani, G.; Nishiyama, Y.; Seeberger, P. H.; Ogawa, Y.; Delbianco, M. Supramolecular Assembly and Chirality of Synthetic Carbohydrate Materials. Angew. Chem., Int. Ed. 2020, 59, 22577-22583.

(29) Zhao, B.; Yang, S.; Deng, J.; Pan, K. Chiral Graphene Hybrid Materials: Structures, Properties, and Chiral Applications. Adv. Sci. 2021, 8, 2003681.

(30) Nemati, A.; Shadpour, S.; Querciagrossa, L.; Mori, T.; Zannoni, C.; Hegmann, T. Highly Sensitive, Tunable Chirality Amplification through Space Visualized for Gold Nanorods Capped with Axially Chiral Binaphthyl Derivatives. ACS Nano 2019, 13, 10312-10326. 